\title{
1 Irradiance modulates thermal niche in a previously undescribed low-light and cold-
}

2 adapted nano-diatom

3 Joshua D. Kling ${ }^{1,2}$, Kyla J. Kelly ${ }^{1}$, Sophia Pei ${ }^{1}$, Tatiana A. Rynearson ${ }^{3}$, David A. Hutchins ${ }^{1}$

$5{ }^{1}$ Department of Biological Sciences, University of Southern California, Los Angeles, CA, USA

$6 \quad{ }^{2}$ QB3-Berkeley, University of California Berkeley, Berkeley, CA, USA

$7 \quad{ }^{3}$ Graduate School of Oceanography, University of Rhode Island, Narragansett, RI, USA

$9 \quad \underline{\text { Abstract }}$

Diatoms have well-recognized roles in fixing and exporting carbon and supplying energy

11 to marine ecosystems, but only recently have we begun to explore the diversity and importance

12 of nano- and pico-diatoms. Here we describe a small $(\sim 5 \mu \mathrm{m})$ diatom from the genus

13 Chaetoceros Isolated from a wintertime temperate estuary $\left(2^{\circ} \mathrm{C}\right.$, Narragansett Bay, RI), with a

14 unique obligate specialization for low-light environments $\left(<120 \mu \mathrm{mol}\right.$ photons $\left.\mathrm{m}^{-2} \mathrm{sec}^{-1}\right)$. This

15 diatom exhibits a striking interaction between irradiance and thermal responses whereby as temperatures increase, so does its susceptibility to light stress. Historical 18S rRNA amplicon

17 data from our study site show this isolate was abundant throughout a six-year period, and its

18 presence strongly correlates with winter and early spring months when light and temperature are

19 low. Two ASVs matching this isolate had a circumpolar distribution in Tara Polar Ocean Circle

20 samples, indicating its unusual light and temperature requirements are adaptations to life in a

21 cold, dark environment. We expect this isolate's low light, psychrophilic niche to shrink as

22 future warming-induced stratification increases both light and temperature levels experienced by

23 high latitude marine phytoplankton. 
24 Introduction:

Photosynthesis by marine phytoplankton is responsible for $50 \%$ of the global conversion

26 of inorganic $\mathrm{CO}_{2}$ to organic biomass. These photosynthetic protists also perform multiple

27 ecosystem services (1-3), making them essential to study in the context of global climate change

28 (4). Current projections suggest a rise in mean sea surface temperature (SST) of $4{ }^{\circ} \mathrm{C}$ by 2100 (5)

29 which could have large impacts on the physiology and composition of these microbial

30 photosynthetic communities, and their ability to sequester carbon (6). However, making

31 predictions about warming effects on phytoplankton can be difficult, because of their still

32 relatively underexplored biological diversity. This is especially true of small $(<5 \mu \mathrm{m})$ nano and

33 picoplankton, which prior to the advent of next-generation sequencing have been routinely

34 under-sampled by microscopic techniques $(7,8)$. For example, the smallest known diatom genus

35 (Minidiscus) was recently shown to be capable of forming dense blooms, and similar very small

36 diatom groups are now recognized as being globally abundant (8).

Although our knowledge of phytoplankton diversity is expanding, it is an open question how much functional thermal diversity exists within this observed phylogenetic diversity. For instance, phytoplankton communities can typically sustain growth well beyond current mean temperatures. However, excursions above historical thermal maximum thresholds can cause

41 major community restructuring (9), and so affect biogeochemistry (10,11). Lineage-specific

42 predictions of temperature responses have often been based on just a handful of cultured model

43 isolates, such as the diatom Thalassiosira pseudonana, (12), the coccolithophore Emiliania

44 huxleyii (11), and the diazotrophic cyanobacterium Trichodesmium erythraeum $(13,14)$.

45 However, the model organism approach to understanding resilience to rising temperatures in

46 marine phytoplankton undoubtedly under-samples the potential range of thermal responses. 
47 Furthermore, lab-derived growth rates and other proxies for fitness can be uncertain predictors of

48 ecological success. In situ variations in temperature, light, and nutrients and interspecific

49 interactions (e.g. competitive or trophic interactions) can make it difficult to apply conclusions

50 from lab-based studies to natural communities.

51 In this study we expand our knowledge of nanoplankton diversity relative to temperature

52 and light, by characterizing a previously unrecognized nanodiatom from a temperate estuary

53 belonging to the genus Chaetoceros. We place its physiology and life history into an

54 environmental context by combining laboratory experiments with a wealth of taxonomic and

55 ecological time series data spanning six years. In addition, we explored the Tara Oceans dataset

56 to explore its global distribution. We report that this Chaetoceros isolate exhibits a unique

57 physiological relationship between light and temperature, which skews its abundance strongly

58 towards periods of low light and cold temperatures. This wintertime specialist is thus potentially

59 vulnerable to the warmer conditions expected at mid- and high-latitudes with continuing climate

60 change.

61 Methods:

\section{Isolation and Culturing}

63 This diatom was isolated from water collected at the Narragansett Bay Time Series (15)

64 site (latitude 41.47, longitude -71.40) in March, 2018. SST was $2^{\circ} \mathrm{C}$ at the time of collection.

65 Surface water was prefiltered through a $100 \mu \mathrm{m}$ mesh to remove large grazers, and then sorted at

66 the University of Rhode Island Graduate School of Oceanography using a BD Influx flow

67 cytometer (San Jose, CA, USA). Cells approximately $5 \mu \mathrm{m}$ and smaller with chlorophyll a

68 fluorescence were sorted into 96 well plates containing natural seawater amended with nutrients

69 following the recipe for $\mathrm{F}$ media diluted to F/20 (16). Wells showing positive growth over time 
were transferred to new media while gradually increasing nutrients to $F / 2$ concentrations in

71 Aquil artificial seawater (17). Both initial isolates and stock cultures were maintained at $4{ }^{\circ} \mathrm{C}$ and

$7230 \mu \mathrm{mol}$ photons $* \mathrm{~m}^{-2} * \mathrm{sec}^{-1}$ of cool-white fluorescent light on a 12:12 L:D cycle, and diluted

73 biweekly with fresh medium. Several dozen morphologically identical strains were collected, all

74 with an apparent sensitivity to light (data not shown). A single isolate strain was selected at

75 random and used for all subsequent experiments.

76 Temperature and Light Assays

77 All culture work was done in climate controlled walk-in incubators under cool white

78 fluorescent lights. Light levels were verified with daily measurements using a LI-250A light

79 meter (LI-COR Biosciences, Lincoln, NE, USA). Cultures were kept in triplicate $15 \mathrm{ml}$

80 polystyrene culture vials, and temperatures for all experiments were set using a series of water

81 baths, each with its own thermostat, heater, and cooling element. Replicates were kept in

82 exponential phase by diluting cultures with sterile medium when biomass reached a

83 predetermined threshold. Cultures were acclimated to each combination of irradiance $(15,30,50$,

$8460,70,100$, and $120 \mu \mathrm{mol}$ photons $\left.\mathrm{m}^{-2} \mathrm{sec}^{-1}\right)$ and temperature $(2,8,12,14,16,18,20,22,24,26$

$85{ }^{\circ} \mathrm{C}$ ) for two weeks.

After acclimation, growth rates were determined daily using in vivo fluorescence on a

87 Turner AU-10 fluorometer (Turner Designs Inc., Sunnyvale, CA, USA) for an additional seven

88 to ten days. In vivo fluorescence was used as a proxy for photosynthetic biomass, because it

89 allowed efficient daily measurements of a large number of simultaneously maintained cultures

90 (as many as 90 at a time). All well-acclimated replicates were kept in the same nutrient

91 conditions, and growth rates were calculated from in-vivo fluorescence of each individual

92 replicate measured relative to itself over time (9,18-21). Although In-vivo fluorescence was 
93 never used to compare biomass across different light and temperature treatments, in a pilot study

94 we observed that fluorescence and cell counts increased linearly even across different light and

95 temperature treatments (Figure S1).

96 Specific growth rates were calculated with the GrowthTools R package

97 (DOI:10.5281/zenodo.3634918) using the slope of a regression line fit to the log of these data

98 (20). Growth rates for cultures acclimated to $16^{\circ} \mathrm{C}$ were calculated for seven light intensities. In

99 addition, we measured growth rates versus each temperature in cultures acclimated to irradiances

100 of 15,30 , or $50 \mu \mathrm{mol}$ photons $\mathrm{m}^{-2} \mathrm{sec}^{-1}$. GrowthTools (DOI:10.5281/zenodo.3634918) was used

101 again to calculate thermal performance curves (TPCs) using the Eppley-Norberg model $(22,23)$.

102 For the thermal curve done under $15 \mu \mathrm{mol}$ photons $\mathrm{m}^{-2} \mathrm{sec}^{-1}, 4{ }^{\circ} \mathrm{C}$ was used as the lowest

103 temperature instead of $2{ }^{\circ} \mathrm{C}$.

In addition to acclimated growth experiments, we exposed the cultures to short-term

105 doses of extreme light levels on the order of hundreds of $\mu \mathrm{mol}$ photons $\mathrm{m}^{-2} \mathrm{sec}^{-1}$, similar to

106 published diatom light-stress experiments $(24,25)$. For these experiments we used $\sim 638 \mu$ mol

107 photons $\mathrm{m}^{-2} \mathrm{sec}^{-1}$, which approximated the highest value measured in a 50-year dataset of surface 108 irradiance from the Narragansett Bay Time Series

109 (https://web.uri.edu/gso/research/plankton/data/). Triplicate cultures acclimated to either $4{ }^{\circ} \mathrm{C}$ or $11016{ }^{\circ} \mathrm{C}$ and $30 \mu \mathrm{mol}$ photons $\mathrm{m}^{-2} \mathrm{sec}^{-1}$ were exposed to this extreme light level for one, three, or

111 six hours, and compared to triplicate cultures that were not exposed (negative control), or were

112 continuously exposed (positive control). After the exposure period, cultures were moved back to $11330 \mu \mathrm{mol}$ photons $\mathrm{m}^{-2} \mathrm{sec}^{-1}$ and fluorescence was recorded twice daily over three days of a 12:12

114 hour L:D cycle.

\section{Sequencing}


For sequencing, $200 \mathrm{ml}$ of dense culture was filtered onto a $0.22 \mu \mathrm{m}$ polyethersulfone

117 Whatman Nuclepore filter (GE Healthcare, Chicago, IL, USA), flash frozen with liquid nitrogen

118 and stored at $-80^{\circ} \mathrm{C}$. DNA was extracted using a DNEasy Power Water kit (Qiagen, German

119 Town, MD, USA), and prepared for sequencing using the Nextera DNA Flex Library Prep kit

120 (Illumina, San Diego, CA, USA). Sequencing was done at the University of Southern

121 California's Genome Core on a Illumina Nextseq 550. Raw sequence data was quality checked

122 using Fastqc (https://www.bioinformatics.babraham.ac.uk/projects/fastqc/, v. 0.11.8) and

123 Multiqc (26)(v. 1.6) and low quality bases were removed with Trimmomatic (27)(v. 0.38). To

124 recover 18S rRNA gene sequences, we used Bowtie2 (28)(v. 2.3.5) to map all reads to a dataset

125 of 200 complete or nearly complete (>1000 bp) Chaetoceros $18 \mathrm{~S}$ rRNA gene sequences

126 downloaded from NCBI. Reads that mapped even once were recovered using Seqtk

127 (https://github.com/lh3/seqtk, v. 1.3) and assembled with SPAdes (29)(v. 3.11). To identify our

128 isolate, full length copies of the 18S rRNA gene sequence were downloaded from NCBI for 25

129 distinctly named species of Chaetoceros. The pennate diatom Pseudo-nitzschia australis was

130 included as an outgroup. All sequences were aligned using Muscle (30)(v. 3.8.31), the alignment

131 trimmed using trimAL (31)(v. 1.4.15), and FastTree (32)(v. 2.1.10) was used to construct a

132 phylogenetic tree.

Six years of amplicon sequencing data from our study site using diatom-specific primers

134 matching the V4 hypervariable region of the 18S rRNA gene (33) were obtained from (15). Raw

135 sequence data were downloaded from NCBI (PRJNA327394) and quality filtered as for the

136 Illumina sequencing. Quality-controlled reads were merged and denoised into Amplicon

137 Sequence Variants (ASVs) using DADA2 (Callahan et al. 2016, v. 1.14.0). BLAST (McGinnis 
138

139

140

141

142

143

144

145

146

147

148

149

150

151

152

153 diatom using BLAST $(35,37)$. In addition, we downloaded the Tara Polar dataset and analyzed

and Madden 2004, v. 2.9.0) was used to identify ASVs that matched the V4 rRNA gene sequence from the full length sequence assembled from our genomic data.

We also mined years of observational data to put the occurrence of our isolate ASV in Narragansett Bay into a long-term temperature and irradiance context. Sea surface temperature (SST) data matching the amplicon sequencing data were downloaded from the Narragansett Bay Time-series website (https://web.uri.edu/gso/research/plankton/data/). Dates without SST measurements from the time-series dataset were supplemented by SST data from the National Data Buoy Centers station QPTR1 - 8454049 at nearby Quonset Point (https://www.ndbc.noaa.gov/station_page.php?station=qptr1). Irradiance data for Narragansett Bay were downloaded from the National Research Reserve System's Central Data Management Office website (https://cdmo.baruch.sc.edu/dges/) for station NARPCMET. To avoid overinflating potential correlations between changing relative abundance within the diatom community (caused by differences in $18 \mathrm{~S}$ rRNA gene copy number) and environmental factors, we used one percent relative abundance as a threshold and calculated the changing probability of an observation being above this threshold under different conditions. For instance, if this diatom had a relative abundance $>1 \%$ in half of a group of samples then its probability of detection was 0.5 .

We utilized the Tara Oceans V9 amplicon dataset (36) to understand the distribution of this isolate beyond Narragansett Bay. Sequence data from low- and mid- latitudes previously analyzed and resolved into ASVs using DADA2 were screened for the presence of this isolate all amplicon sequencing data for these Arctic Ocean surface samples collected on filters with a pore size $<5 \mu \mathrm{m}$. Reads were denoised following the methods of (37). 
Statistics and Data Availability

All statistics for analyzing these data and graphic visualizations were done using $\mathrm{R}$ made publicly available at https://figshare.com/projects/nanodiatom_temp_light/74283.

169 Sequence data can be found on NCBI under the SRA accession PRJNA608686 (raw Illumina 170 reads) and MT742785 (assembled 18S rRNA sequence).

171 Results:

$17218 S$ rRNA resolved taxonomy

173 Short read sequencing produced nine million 150bp paired-end Illumina reads. Mapping 174 reads to 200 full length Chaetoceros spp. 18S sequences and assembling all mapped reads 175 produced a single contig 1812 bp long. When BLASTed against the nt database, excluding all 176 non-cultured isolates, this assembled $18 \mathrm{~S}$ rRNA gene sequence was the closest match to $C$. $c f$. 177 wighamii strain BH65_48, with $99.8 \%$ identity across $92 \%$ of the sequence (accession

178 KY980353.1). Unfortunately, isolation information was not available for this strain; however, 179 the next closest match was to another C. cf. wighamii strain from the Roscoff Culture Collection 180 (RCC3008, KT860959.1) at 100\% identity across $90 \%$ of the query. This strain was isolated 181 from the coastal Baltic Sea in 2010 at $4{ }^{\circ} \mathrm{C}$, and was maintained at $50 \mu \mathrm{mol}$ photons $\mathrm{m}^{-2} \mathrm{sec}^{-1}$, 182 similar to our isolate. Aligning 25 full length 18S sequences for named Chaetoceros species

183 (Table S1) allowed us to construct a high quality phylogenetic tree (average maximum likelihood 
$184=0.91$ ) of this genus (Figure 1). C. cf. wighamii was the closest branching sequence, followed by temperate isolates $C$. throndsenii from the Gulf of Naples (93.6\% ID and 96\% coverage), and $C$. lorenzianus (94.1\% ID and 90\% coverage) and C. constrictus (93\% ID and 94.3\% coverage)

187 from Las Cruces, Chile.

When grown at $16^{\circ} \mathrm{C}$, this diatom isolate had an asymmetric response to increasing light

$191 \mu \mathrm{mol}$ photons $\left.\mathrm{m}^{-2} \mathrm{sec}^{-1}\right)$ the specific growth rate was only $0.13 \mathrm{day}^{-1}( \pm 0.01)$, when irradiance $( \pm 0.04), 0.20( \pm 0.02)$, and $0.18 \mathrm{day}^{-1}( \pm 0.04)$ respectively, and they were statistically

197 indistinguishable from each other $(p>0.05)$. Growth rates of our Chaetoceros isolate dropped

198 significantly to only $0.10 \pm 0.01$ day $^{-1}$ at $100 \mu \mathrm{mol}$ photons $\mathrm{m}^{-2} \mathrm{sec}^{-1}(p<0.05)$, and growth was 199 negative $\left(-0.06\right.$ day $\left.^{-1}, \pm 0.01\right)$ when light levels were increased to $120 \mu \mathrm{mol}$ photons $\mathrm{m}^{-2} \mathrm{sec}^{-1}$,

200 leading to eventual cell death (Figure 2).

\section{Thermal Curves}

203 thermal performance curves (TPCs) at three different irradiance levels $(15,30$, and $50 \mu \mathrm{mol}$

204 photons $\mathrm{m}^{-2} \mathrm{sec}^{-1}$ ) are shown in Figure 3a and Table 1. Here we abbreviate the light treatments as

205 low, optimal, and high light. Using a repeat measures ANOVA, each of these TPCs was

206 significantly different from each other (Figure 3a, $p<0.001$ ). Comparing values obtained from 
the Eppley-Norberg model, the full range of growth temperatures (the thermal niche width) was broadest at optimal and low light $\left(27.0\right.$ and $25.7^{\circ} \mathrm{C}$, respectively), while the high light niche width was only $23.2{ }^{\circ} \mathrm{C}$ (Table 1 ). The difference in modelled niche width compared to optimal 210 light was manifested as a $1.3{ }^{\circ} \mathrm{C}$ decrease in the upper temperature limit (Tmax) under low light

$211\left(23.7^{\circ} \mathrm{C}\right)$, and as a $3.5^{\circ} \mathrm{C}$ increase in the lower temperature limit (Tmin), under high light $(1.5$

$212{ }^{\circ} \mathrm{C}$ ) (Table 1). The model predicted that the optimal growth temperature (Topt) would be higher 213 at $30 \mu \mathrm{mol}$ photons $\mathrm{m}^{-2} \mathrm{sec}^{-1}\left(17.2^{\circ} \mathrm{C} \pm 0.86\right.$, Fig $3 \mathrm{~b}$, Table 1$)$. The Topt decreased under both

214 low and high light, and although it fell farther in low light, both low and high light Topts were 215 within one standard deviation of each other $\left(13.7 \pm 0.87\right.$ and $15.2 \pm 1.17^{\circ} \mathrm{C}$ respectively). The maximum specific growth rate ( $\mu \mathrm{Max})$ across these TPC models was estimated to be highest

217 under optimal light $\left(0.28\right.$ day $\left.^{-1} \pm 0.02\right)$ and high light $\left(0.25\right.$ day $^{-1} \pm 0.02$, Figure $3 \mathrm{c}$, Table 1$)$.

218

\section{Response to Extreme Light Stress}

In addition to considering how this diatom responded when acclimated to constant light and temperature conditions, we also assessed how it responded to pulses of extreme light at 4 and $16{ }^{\circ} \mathrm{C}$. At both temperatures, constant exposure to extreme light levels $\left(638 \mu \mathrm{mol}\right.$ photons $\mathrm{m}^{-}$ ${ }^{2} \mathrm{sec}^{-1}$ ) was lethal. At $4{ }^{\circ} \mathrm{C}$ the constant extreme light treatment (positive control) fluorescence decreased steadily until it reached the lower limit of detection at the very end of this experiment (Figure 4a); however, at $16^{\circ} \mathrm{C}$ fluorescence reached the lower limit after just 24 hours (Figure 4c), 3.3x faster (Figure $4 \mathrm{~b} \& \mathrm{~d}$ ). At the lower temperature this Chaetoceros isolate maintained positive growth even after being exposed to extreme light for six hours, although exposure for both three and six hours significantly decreased the growth rate compared to cultures never exposed to extreme light (negative control, $p<0.05$ ). Similarly, measured growth rates were significantly lower after three- and six-hour exposures compared with the negative control. Cells 
230

231

232 lethal.

233

234

235

236

237

238

239

240

241

242

243

244

245

246

acclimated to $16^{\circ} \mathrm{C}$ also had significantly lower growth rates compared to unexposed cultures $(p$ $<0.05)$; however, unlike at the colder temperature, exposure to extreme light for six hours was

\section{Environmental Amplicon Data}

Before using the assembled 18S rRNA sequence to look for this diatom in available amplicon sequencing data from Narragansett Bay, we first confirmed that there was enough diversity within the V4 hypervariable region across this genus to distinguish our isolate from other Chaetoceros spp.. Using aligned V4 regions of the same sequences in Figure 1 we were able to construct a phylogenetic tree (Figure S2) that separated our isolate from other members of the genus. Processing sequencing data from Narragansett Bay resulted in 5170 distinct amplicon sequence variants (ASVs); however, only 20 of these had an average relative abundance greater than one percent of recovered amplicons across the data set. When BLASTed, the most common diatom genera were Thalassiosira (eight), Skeletonema (four), and Minidiscus (two)(Table S2). These are consistent with previous observations at Narragansett Bay, where

Thalassiosira and Skeletonema often dominate the diatom community $(15,40)$. Of these 20 most abundant recovered ASVs, one was a perfect match (100\% ID and $100 \%$ coverage) to the $18 \mathrm{~S}$ rRNA gene sequence of our isolate.

This ASV was greater than one percent of the total recovered amplicons in 28 of the 80 samples. It had a $\sim 0.5$ probability of detection in samples in January, February, and March (Figure 5a). This probability peaked at 0.83 in April, before decreasing to 0.43 again in May. In June through August the probability of detection was $\sim 0.2$ and dropped to zero in September and October, before rising again to 0.5 in November and December (Figure 5a). Across all 80 samples it comprised on average $4.1 \%$ of the relative diatom sequence reads per sample, with a 
maximum of $76.8 \%$ of recovered amplicons on May $28^{\text {th }}, 2010$ (Figure S3). From the available

254 observational data we were not able to associate this isolate with major phytoplankton blooms in

255 Narragansett Bay. In this dataset, chlorophyll a concentrations were greater than $10 \mu \mathrm{g} / \mathrm{L}$ in 10

256 different samples. The ASV matching our Chaetoceros isolate was only detected above the one

257 percent relative abundance in three of these high chlorophyll a events, but in each of these

258 samples where it was detected it never made up more than $1.5 \%$ of the recovered amplicons.

To examine the thermal and light niches of the ASV matching our Chaetoceros isolate,

260 we split temperatures into 13 two-degree bins from 0 to $26{ }^{\circ} \mathrm{C}$. The isolate was present in

261 Narraganset Bay from 0-24 deg C but occurred more frequently at temperatures $<12{ }^{\circ} \mathrm{C}$. At

262 these cooler temperatures the probability of observing this ASV was on average $0.52( \pm 0.19)$, but

263 dropped to $0.11( \pm 0.11)$ above $12{ }^{\circ} \mathrm{C}$, almost five times lower. The distribution of light exposure

264 readings throughout the dataset was largely 10 to 40 moles photons $\mathrm{m}^{-2}$ day $^{-1}$, so measurements

265 within this range were grouped in increments of 5 moles photons $\mathrm{m}^{-2}$ day $^{-1}$, with readings outside

266 this distribution recorded as $<10$ and $>40$ moles photons $\mathrm{m}^{-2}$ day $^{-1}$, respectively. The probability

267 of detection was approximately equal at $\sim 0.3$ for all total irradiance levels $>10$ moles photons $\mathrm{m}^{-2}$

268 day $^{-1}$; however, when total irradiance was $<10$ moles photons $\mathrm{m}^{-2}$ day $^{-1}$, the probability of

269 detection nearly tripled to 0.9 (Figure 5c).

270 In order to assess this isolate's geographic distribution beyond Narragansett Bay, we

271 accessed amplicon data from the Tara Oceans dataset. Prior to analysis, a phylogenetic tree was

272 made using the V9 region of the 18S rRNA genes used in Figure 4 to show that these primers

273 were able to differentiate our isolate from other Chaetoceros spp. (Figure S4). This isolate was

274 not detected in previously published amplicon data from the Tara Oceans project, which largely

275 cover tropical and temperate latitudes (37). However, two ASVs that were $100 \%$ match across 
$276>90 \%$ of the sequence were detected in surface waters at 8 of 16 stations of the high-latitude

277 Tara Oceans Polar Circle sampling (Figure 6). Both ASVs were found at the same stations with

278 approximately equal relative abundance, and thus their results are reported together.

279 Interestingly, they were mostly detected at stations where the relative abundance of diatom

280 amplicons was comparatively low (Figure S5).

281 Discussion:

In this study we describe a recently isolated nanodiatom with $18 \mathrm{~S}$ rRNA gene sequence

283 similarity to diatoms identified as Chaetoceros $c f$. wighamii in NCBI. In laboratory culture

284 experiments this isolate showed a strong sensitivity to light, unusual for planktonic diatoms. This

285 sensitivity impacted (and was impacted by) temperature, and broadly explains this diatom's

286 seasonal distribution across multiple years of $18 \mathrm{~S}$ rRNA gene amplicon data from its isolation

287 location. Despite this seemingly unusual physiology, this Chaetoceros $s p$. was detected across

288 the samples taken during the Tara Oceans Polar Circle Expedition. This suggests its physiology

289 may not in fact be that unusual, but rather part of a broader adaption to high latitude waters.

A preference for low light levels for growth is not necessarily uncommon in marine

291 phytoplankton. For instance, Prochlorococcus, a dominant unicellular marine

292 picocyanobacterium in the oligotrophic gyres, has well-defined low- and high-light ecotypes

293 occupying deep and surface layers of the euphotic zone, respectively (41-43). Studies on low

294 light Prochlorococcus have reported upper light limits that are similar to those we describe here

295 for our diatom isolate (41). Even among diatoms, adaptations to low light levels have been

296 reported in species living in benthic environments (44). However, centric diatoms are not

297 typically considered to be part of the benthic community (although dormant resting stages can be 
isolate is an adaptation to deeper layers of the photic zone, similar to low-light Prochlorococcus, but its consistent presence even in open ocean surface water samples from high latitudes does not support either conclusion. Another possibility is this diatom could be adapted to living under sea ice, where active photosynthesis can be maintained below $5 \mu \mathrm{mol}$ photons $\mathrm{m}^{-2} \mathrm{sec}^{-1}(48)$; however, this seems unlikely as temperate environments such as Narragansett Bay typically do

304 not freeze over during the winter (49). spring progresses into summer and day length and angle of solar incidence increase. Our data temperature and light. For instance, in six years of amplicon data it was detected in $\sim 45-80 \%$ of

312 the samples taken between November and April, when temperatures are low and the days are

313 relatively short. The frequency of detection in May could be explained by the fact that although

314 day length is increasing, the waters in Narragansett Bay are still colder than summer and early

315 fall conditions (mean $\left.=15.5 \pm 2.7^{\circ} \mathrm{C}\right)$. In the summer months (June through September) it was

316 only detected 4 times. That it was detected at all during these warmer, brighter months suggests

317 there may be additional environmental factors (such as nutrient availability) controlling its

318 distribution. Interactions between light and temperature also explain why observations of this

319 diatom in situ typically occur at temperatures well below the range of optimal growth

320 temperatures predicted by our TPC models $\left(13.7-17.2^{\circ} \mathrm{C}\right)$. It should be stated however that only 
3215 observations were made between $10-14^{\circ} \mathrm{C}$, and it could be that more samples in this range

322 would change the frequency of detection.

It is interesting to consider how these experimental and seasonal data may inform this

324 diatom's distribution in the broader ocean. For instance, although this Chaetoceros sp. was

325 detected in half of the Tara Oceans Polar Circle samples, these were collected between May and

326 October. Based on the seasonality depicted in data from Narragansett Bay this is when we would

327 expect its abundance to be lowest. Consequently, they may be even more abundant in polar

328 waters than observed here. SST across theses samples was low (2.56 average $\left.\pm 3.7^{\circ} \mathrm{C}\right)$ compared

329 to Narragansett Bay; however, irradiance was likely much higher due to the near constant

330 daylight experienced during the polar summer. In culture experiments we observed that this

331 diatom's growth rate decreased at similar temperatures when light was supraoptimal (Figure 3a)

332 which suggests that its physiology could be an adaptation to the cold and low-light conditions

333 found during early spring months in the North Atlantic $(51,52)$. Future studies using molecular

334 methods to look at the composition of early spring bloom may show that this diatom contributes

335 significantly to primary production at high latitudes.

Although this study documents this diatom's singular low-light niche, more work will be

337 needed to investigate the mechanisms involved. For photosynthetic organisms, an accumulation

338 of deleterious reactive oxygen species (ROS) in the cell (in particular the chloroplast) is often

339 seen following exposure to extreme irradiance $(53,54)$. Under low light conditions, many

340 diatoms photo-acclimate by increasing the size of their chloroplasts and the number of

341 photosystems and antenna pigments they contain, in order to increase photon capture $(55,56)$. It

342 could be that our low-light Chaetoceros has a limited ability to adjust its photosynthetic energy

343 acquisition systems when exposed to high light, causing a harmful buildup of ROS. Similarly, 
344 variation in the xanthophyll cycle $(57,58)$ and production of ROS-scavenging antioxidants $(58)$

345 could contribute to this planktonic diatom's unusual physiology.

Future work should also consider the effect of light spectral quality on the irradiance and

347 temperature interactions described here. In aquatic environments not only is the total irradiance

348 variable, but also the availability of specific wavelengths. Shorter wavelength blue light has

349 more energy, and thus penetrates farther into the water column than longer red wavelengths.

350 Phytoplankton associated with low light environments such as deep water or beneath sea ice are

351 often specialized for utilizing these higher energy wavelengths $(59,60)$. Blue light has also been

352 suggested to trigger germination in the resting stages of marine diatoms, and has been associated

353 with upregulation of proteins associated with photoprotection in Phaeodactylum tricornatum

$354(61,62)$. At higher latitudes and during the winter, solar elevation is lower compared to low

355 latitudes or during the summer. This results in a lower angle of incidence, which causes more

356 light to be reflected from the ocean's surface; however, this process is skewed towards longer

357 wavelengths, which are preferentially reflected (63). The implication is that phytoplankton at

358 higher latitudes or during the winter season experience more blue light relative to red light. It

359 would be interesting to test whether these diatoms experience the same light sensitivity when

360 grown under blue light as white light (as in this study).

361 The interactive effects of light and temperature on this diatom's growth in the lab and

362 pattern of abundance in situ raise interesting questions about how marine phytoplankton will

363 respond to rising temperatures associated with climate change. It is broadly suggested that

364 organisms at high latitudes exist at temperatures well below their thermal optima, and therefore

365 rising temperatures will be advantageous, increasing their growth rate $(23,64)$. The average

366 temperature at Narragansett Bay in the five years of temperature data accompanying this 
amplicon dataset is $12.4^{\circ} \mathrm{C}$, below the optimal temperatures predicted by our three TPC models.

However, because of the strong regulation of thermal niche by light level in this Chaetoceros $s p$.

it could be that this isolate will not fare better with rising temperatures, as warmer conditions increase its susceptibility to light stress. In a shallow $(8 \mathrm{~m})$, well mixed estuary such as

371 Narragansett Bay this interaction between light and temperature may in fact shrink the range of

372 months where growth of this diatom is feasible. For instance, it is frequently (appearing in $>40 \%$

373 of the samples) observed as late in the year as April and May, where day length is longer and

374 solar elevation higher than during the winter months. Rising temperatures during those months

375 may be harmful, increasing the diatom's susceptibility to light stress; although it may also be that

376 rising temperatures will be advantageous during winter months (e.g. December to March) when

377 light levels are seasonally low.

In the open ocean, this diatom's sensitivity to light may disadvantage it in a warmer

379 future. Current models predict a shoaling of the thermocline at high latitudes, increasing light

380 exposure by trapping phytoplankton closer to the surface (65). This is expected to increase

381 overall photosynthetic growth, as high latitude phytoplankton are often considered light limited

382 (66); however, the Chaetoceros isolate described here which was observed across the polar circle

383 challenges this paradigm. Future work at high latitudes should further investigate the abundance

384 of this diatom (especially during early spring bloom conditions) in order to better predict how

385 climate change will impact phytoplankton communities in these regions.

386 Our study highlights one facet of the largely unrecognized but almost limitless diversity

387 that exists in marine phytoplankton communities. It is fascinating that a planktonic diatom with

388 such a specialized light and temperature niche was discovered at the longest running

389 phytoplankton timeseries in existence, and that despite its specialization it is one of the more 
390 common diatoms at this well-studied site. This work also shows that light and temperature can

391 interact to define a thermal niche. Even in species that thrive at comparatively high light levels,

392 changes in light could similarly impact their response to changes in temperature and influence

393 how they will fare in a warming ocean. Future studies should consider high light as an interactive

394 variable along with other co-stressors such as elevated temperatures and $\mathrm{CO}_{2}$ when predicting

395 how phytoplankton will respond to global change.

396 Acknowledgements:

397 We would like to thank Amanda Montalbano, Meghan Phan and Roxanna Andrade for 398 assistance with preliminary culture work and Daniel Campo for help with library prep and

399 Illumina sequencing. Funding was provided by National Science Foundation (NSF) grants

400 OCE1538525 and OCE1638804 to DAH, and OCE1638834 to TAR. Part of this research was

401 conducted using the University of Rhode Island's Marine Science Research Facility, supported

402 by NSF EPSCoR awards 1004057 and 1655221. 


\section{$\underline{\text { References: }}$}

406 1. Field CB, Behrenfeld MJ, Randerson JT, Falkowski P. Primary production of the biosphere: Integrating terrestrial and oceanic components. Science. 1998;281(5374):23740.

2. Smith S V, Hollibaugh JT. Coastal metabolism and the oceanic organic carbon balance. Rev Geophys. 1993;31(1):75-89.

411 3. Lyle M. Climatically forced organic carbon burial in equatorial Atlantic and Pacific Oceans. Nature. 1988;335(6190):529-32.

413 4. Hutchins DA, Jansson JK, Remais J V, Rich VI, Singh BK, Trivedi P. Climate change microbiology-problems and perspectives. Nat Rev Microbiol. 2019;17(6):391.

415 5. Pachauri RK, Allen MR, Barros VR, Broome J, Cramer W, Christ R, et al. Climate change 2014: synthesis report. Contribution of Working Groups I, II and III to the fifth assessment report of the Intergovernmental Panel on Climate Change. Ipcc; 2014.

418 6. Hutchins DA, Fu F. Microorganisms and ocean global change. Nat Microbiol. 2017;2(6):17058.

420 7. Abad D, Albaina A, Aguirre M, Laza-Martínez A, Uriarte I, Iriarte A, et al. Is metabarcoding suitable for estuarine plankton monitoring? A comparative study with microscopy. Mar Biol. 2016;163(7):149.

423 8. Leblanc K, Quéguiner B, Diaz F, Cornet V, Michel-Rodriguez M, Durrieu De Madron X, et al. Nanoplanktonic diatoms are globally overlooked but play a role in spring blooms and carbon export. Nat Commun.. 2018;9(1):1-12. http://dx.doi.org/10.1038/s41467-01803376-9

427 9. Kling JD, Lee MD, Fu F, Phan MD, Wang X, Qu P, et al. Transient exposure to novel 
high temperatures reshapes coastal phytoplankton communities. ISME J. 2019. http://dx.doi.org/10.1038/s41396-019-0525-6

430 10. Hare CE, Leblanc K, DiTullio GR, Kudela RM, Zhang Y, Lee PA, et al. Consequences of increased temperature and $\mathrm{CO}_{2}$ for phytoplankton community structure in the Bering Sea. Mar Ecol Prog Ser. 2007;352:9-16.

433 11. Feng Y, Warner ME, Zhang Y, Sun J, Fu F-X, Rose JM, et al. Interactive effects of 434 increased $\mathrm{pCO}_{2}$, temperature and irradiance on the marine coccolithophore Emiliania 435 huxleyi (Prymnesiophyceae). Eur J Phycol. 2008;43(1):87-98.

436 12. Berges JA, Varela DE, Harrison PJ. Effects of temperature on growth rate, cell composition and nitrogen metabolism in the marine diatom Thalassiosira pseudonana

439 13. Mulholland MR, Bernhardt PW. The effect of growth rate, phosphorus concentration, and 440 temperature on $\mathrm{N}_{2}$ fixation, carbon fixation, and nitrogen release in continuous cultures of Trichodesmium IMS101. Limnol Oceanogr. 2005;50(3):839-49.

442 14. Fu F-X, Yu E, Garcia NS, Gale J, Luo Y, Webb EA, et al. Differing responses of marine $443 \quad \mathrm{~N}_{2}$ fixers to warming and consequences for future diazotroph community structure. Aquat $444 \quad$ Microb Ecol. 2014;72(1):33-46.

445 15. Rynearson TA, Flickinger SA, Fontaine DN. Metabarcoding Reveals Temporal Patterns of Community Composition and Realized Thermal Niches of Thalassiosira spp. (

447 Bacillariophyceae ) from the Narragansett Bay Long - Term Plankton Time Series. $448 \quad$ Biology. 2020;9:1-19.

449 16. Guillard RRL. Culture of phytoplankton for feeding marine invertebrates. In: Culture of 450 marine invertebrate animals. Springer; 1975. p. 29-60. 
451 17. Sunda WG, Price NM, Morel FMM. Trace metal ion buffers and their use in culture

453 18. Gilstad M, Sakshaug E. Growth rates of ten diatom species from the Barents Sea at different irradiances and day lengths. Mar Ecol Prog Ser. 1990;64:169-73.

455 19. Chen CY, Durbin EG. Effects of $\mathrm{pH}$ on the growth and carbon uptake of marine 456 phytoplankton. Mar Ecol Ser. 1994;109:83.

457 20. Wood AM, Everroad RC, Wingard LM. Measuring growth rates in microalgal cultures. In: Algal culturing techniques. Elsevier Academic Press London. 2005;18:269-88.

459 21. Ichimi K, Kawamura T, Yamamoto A, Tada K, Harrison PJ. Extremely high growth rate of the small diatom Chaetoceros salsugineum isolated from an estuary in the Eastern Seto Inland Sea, Japan. J Phycol. 2012;48(5):1284-8.

462 22. Norberg J. Biodiversity and ecosystem functioning: A complex adaptive systems approach. Limnol Oceanogr. 2004;49(4 II):1269-77.

23. Thomas MK, Kremer CT, Klausmeier CA, Litchman E. A global pattern of thermal adaptation in marine phytoplankton. Science. 2012;338(6110):1085-8.

24. Dong H-P, Dong Y-L, Cui L, Balamurugan S, Gao J, Lu S-H, et al. High light stress triggers distinct proteomic responses in the marine diatom Thalassiosira pseudonana.

469 25. Zhu S-H, Green BR. Photoprotection in the diatom Thalassiosira pseudonana: role of 470 LI818-like proteins in response to high light stress. Biochim Biophys Acta (BBA)471 Bioenergetics. 2010;1797(8):1449-57.

472 26. Ewels P, Magnusson M, Lundin S, Käller M. MultiQC: summarize analysis results for 473 multiple tools and samples in a single report. Bioinformatics. 2016;32(19):3047-8. 
474 27. Bolger AM, Lohse M, Usadel B. Trimmomatic: a flexible trimmer for Illumina sequence data. Bioinformatics. 2014;30(15):2114-20.

476 28. Langmead B, Salzberg SL. Fast gapped-read alignment with Bowtie 2. Nat Methods.

$477 \quad 2012 ; 9(4): 357$.

478 29. Bankevich A, Nurk S, Antipov D, Gurevich AA, Dvorkin M, Kulikov AS, et al. SPAdes:

479 a new genome assembly algorithm and its applications to single-cell sequencing. J Comput Biol. 2012;19(5):455-77.

481 30. Edgar RC. MUSCLE: multiple sequence alignment with high accuracy and high throughput. Nucleic Acids Res. 2004;32(5):1792-7.

483 31. Capella-Gutiérrez S, Silla-Martínez JM, Gabaldón T. trimAl: a tool for automated alignment trimming in large-scale phylogenetic analyses. Bioinformatics. 2009;25(15):1972-3.

32. Price MN, Dehal PS, Arkin AP. FastTree: computing large minimum evolution trees with profiles instead of a distance matrix. Mol Biol Evol. 2009;26(7):1641-50.

488 33. Zimmermann J, Jahn R, Gemeinholzer B. Barcoding diatoms: Evaluation of the V4 subregion on the $18 \mathrm{~S}$ rRNA gene, including new primers and protocols. Org Divers Evol. 2011;11(3):173-92.

491 34. Callahan BJ, McMurdie PJ, Rosen MJ, Han AW, Johnson AJA, Holmes SP. DADA2: high-resolution sample inference from Illumina amplicon data. Nat Methods. 2016;13(7):581.

494 35. McGinnis S, Madden TL. BLAST: at the core of a powerful and diverse set of sequence analysis tools. Nucleic Acids Res. 2004;32(suppl_2):W20-5. 
diversity in the sunlit ocean. Science. 2015;348(6237):1261605.

37. Callahan BJ, McMurdie PJ, Holmes SP. Exact sequence variants should replace

operational taxonomic units in marker-gene data analysis. ISME J. 2017;11(12):2639.

500

38. Team RC. R: A Language Environment for Statistical Computing. Vienna, Austria: R

39. Team Rs. RStudio: Integrated Development for R. Boston, MA: RStudio, Inc.; 2016. http://www.rstudio.com/

40. Canesi KL, Rynearson TA. Temporal variation of Skeletonema community composition from a long-term time series in Narragansett Bay identified using high-throughput DNA sequencing. Mar Ecol Prog Ser. 2016;556:1-16.

507 41. Goericke R, Olson RJ, Shalapyonok A. A novel niche for Prochlorococcus sp. in lowlight suboxic environments in the Arabian Sea and the Eastern Tropical North Pacific. Deep Sea Res Part I Oceanogr Res Pap. 2000;47(7):1183-205.

42. Moore LR, Rocap G, Chisholm SW. Physiology and molecular phylogeny of coexisting Prochlorococcus ecotypes. Nature. 1998;393(6684):464-7.

512 43. Johnson ZI, Zinser ER, Coe A, McNulty NP, Woodward EMS, Chisholm SW. Niche partitioning among Prochlorococcus ecotypes along ocean-scale environmental gradients.

515 44. Admiraal W. Influence of light and temperature on the growth rate of estuarine benthic diatoms in culture. Mar Biol. 1976;39(1):1-9.

517 45. Conley DJ, Kilham SS, Theriot E. Differences in silica content between marine and freshwater diatoms. Limnol Oceanogr. 1989;34(1):205-12.

519 46. Ligowski R. Benthic feeding by krill, Euphausia superba Dana, in coastal waters off West 
Antarctica and in Admiralty Bay, South Shetland Islands. Polar Biol. 2000;23(9):619-25.

521 47. McQuoid MR, Godhe A. Recruitment of coastal planktonic diatoms from benthic versus pelagic cells: Variations in bloom development and species composition. Limnol Oceanogr. 2004;49(4):1123-33.

524 48. Seckbach J. Algae and Cyanobacteria in Extreme Environments. Astrobiology. 2007:786.

525 49. Pineda J, DiBacco C, Starczak V. Barnacle larvae in ice: Survival, reproduction, and time to postsettlement metamorphosis. Limnol Oceanogr. 2005;50(5):1520-8.

527 50. Edwards KF, Thomas MK, Klausmeier CA, Litchman E. Phytoplankton growth and the interaction of light and temperature: A synthesis at the species and community level. Limnol Oceanogr. 2016;61(4):1232-44.

530 51. Siegel DA, Doney SC, Yoder JA. The North Atlantic spring phytoplankton bloom and Sverdrup's critical depth hypothesis. Science. 2002;296(5568):730-3.

532 52. Boss E, Behrenfeld M. In situ evaluation of the initiation of the North Atlantic phytoplankton bloom. Geophys Res Lett. 2010;37(18):1-5.

534 53. Wilhelm C, Jungandreas A, Jakob T, Goss R. Light acclimation in diatoms: From phenomenology to mechanisms. Mar Genomics. 2014;16(1):5-15. http://dx.doi.org/10.1016/j.margen.2013.12.003

537 54. Mizrachi A, Graff van Creveld S, Shapiro OH, Rosenwasser S, Vardi A. Light-dependent single-cell heterogeneity in the chloroplast redox state regulates cell fate in a marine diatom. Elife. 2019;8:1-27.

540 55. Rosen BH, Lowe RL. Physiological and Ultrastructural Responses of Cyclotella Meneghiniana (Bacillariophyta) To Light Intensity and Nutrient Limitation. Vol. 20, Journal of Phycology. 1984. p. 173-83. 
543 56. Lepetit B, Goss R, Jakob T, Wilhelm C. Molecular dynamics of the diatom thylakoid membrane under different light conditions. Photosynth Res. 2012;111(1-2):245-57.

545 57. Ruban A, Lavaud J, Rousseau B, Guglielmi G, Horton P, Etienne AL. The super-excess energy dissipation in diatom algae: Comparative analysis with higher plants. Photosynth Res. 2004;82(2):165-75.

58. Cartaxana P, Domingues N, Cruz S, Jesus B, Laviale M, Serôdio J, et al. Photoinhibition in benthic diatom assemblages under light stress. Aquat Microb Ecol. 2013;70(1):87-92.

59. Shimada A, Maruyama T, Miyachi S. Vertical distributions and photosynthetic action spectre of two oceanic picophytoplancters, Prochlorococcus marinus and Synechococcus sp. Mar Biol. 1996;127(1):15-23.

60. Gosselin M, Legendre L, Therriault J, Demers S. Light and nutrient limitation of sea-ice microalgae (Hudson Bay, Canadian Arctic). J Phycol. 1990;26(2):220-32.

61. Shikata T, Nukata A, Yoshikawa S, Matsubara T, Yamasaki Y, Shimasaki Y, et al. Effects of light quality on initiation and development of meroplanktonic diatom blooms in a eutrophic shallow sea. Mar Biol. 2009;156(5):875-89.

558 62. Schellenberger Costa B, Jungandreas A, Jakob T, Weisheit W, Mittag M, Wilhelm C.

Phaeodactylum tricornutum. J Exp Bot. 2013;64(2):483-93. 
566 65. Riebesell U, Rtzinger AK, Oschlies A. Sensitivities of marine carbon fluxes to ocean

567 change. Vol. 106, Proc Nat Acad Sci USA. 2009. p. 20602-9.

568 66. Yun MS, Chung KH, Zimmermann S, Zhao J, Joo HM, Lee SH. Phytoplankton

569 productivity and its response to higher light levels in the Canada Basin. Polar Biol.

$570 \quad 2012 ; 35(2): 257-68$.

571

572 
573 Main Figures and Tables:

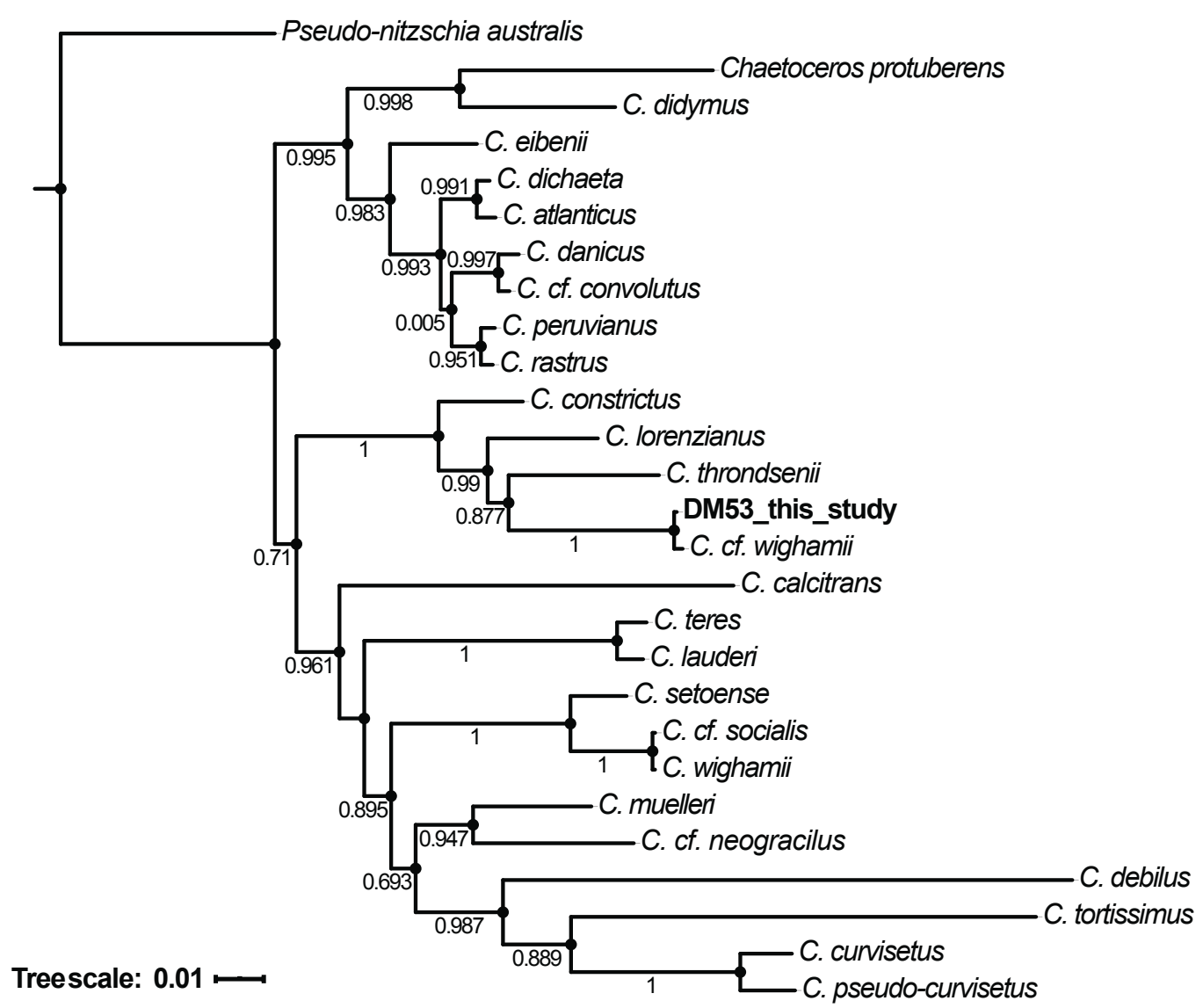

574

575

576

577

578

579
Figure 1: Phylogenetic tree representing the diversity of the diatom genus Chaetoceros constructed using full-length 18S rRNA gene sequences from NCBI. A sequence from the pennate diatom species Pseudo-nitzschia australis is included as an outgroup. The isolate described in this study, DM53, is highlighted in bold. 


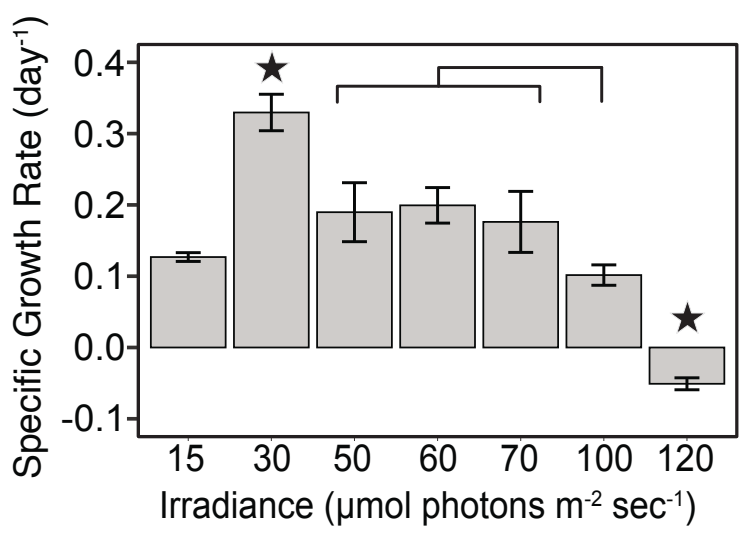

Figure 2: Growth rates at $16{ }^{\circ} \mathrm{C}$ across a range of seven light levels for a novel Chaetoceros sp. isolate. Error bars represent \pm 1 standard deviation. Stars show treatments that are statistically significant $(p<0.05)$ compared to all other treatments via one-way ANOVA. Brackets indicate statistical significance between specific samples. 

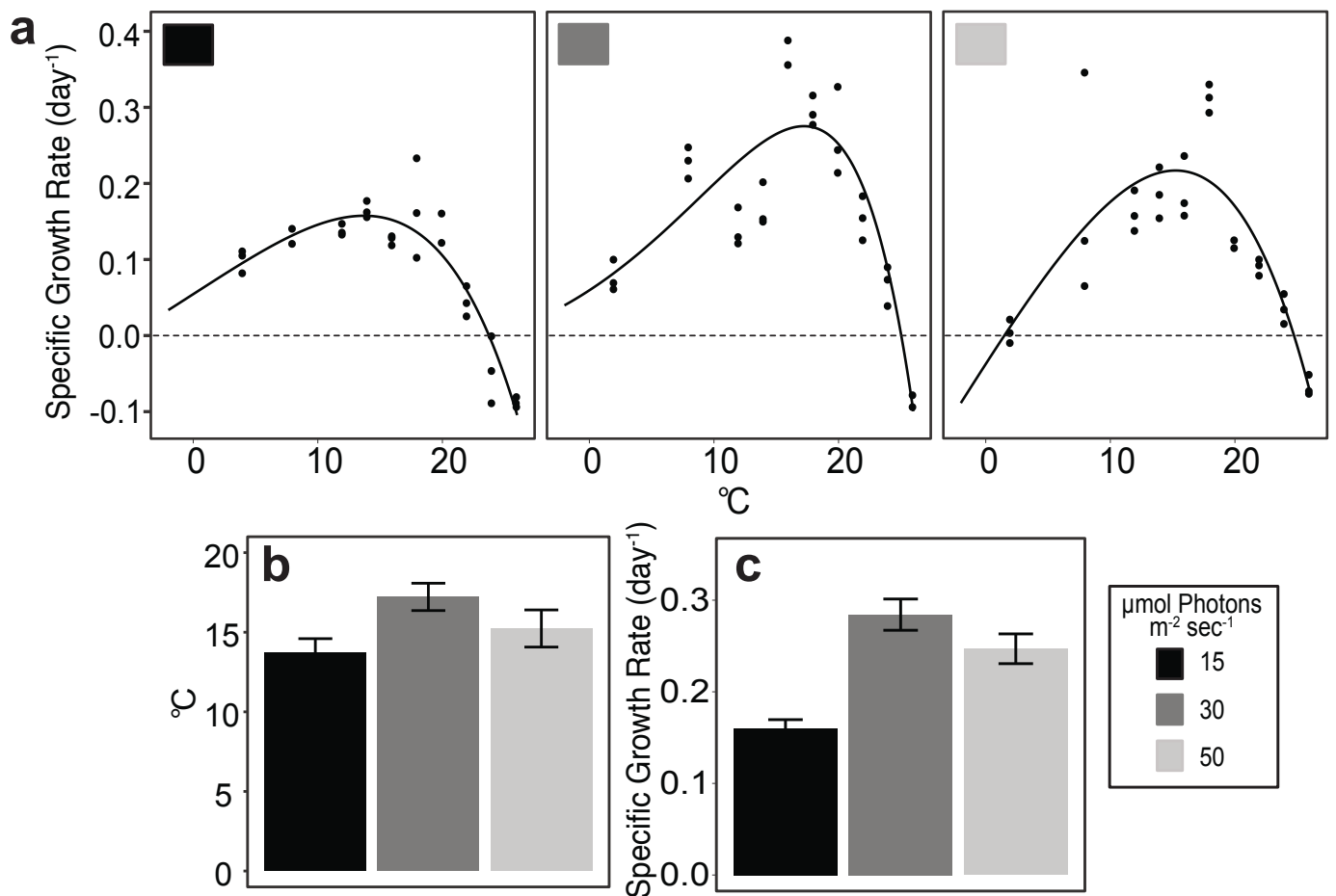

$\mu \mathrm{mol}$ Photons

$\mathrm{m}^{-2} \mathrm{sec}^{-1}$

15

30

50

Figure 3: a) Thermal performance curves across three light levels. For all best-fit curves, $\mathrm{r}^{2}>0.7$ and all are significantly different from each other using repeat measure ANOVA $(p<0.001)$. Differences between light treatments are shown for the b) thermal optimum (Topt) and $\mathbf{c}$ ) maximum growth rate ( $\mu$ Max). Error bars show \pm 1 standard deviation within the modelled optimal temperatures and growth rates for each. 

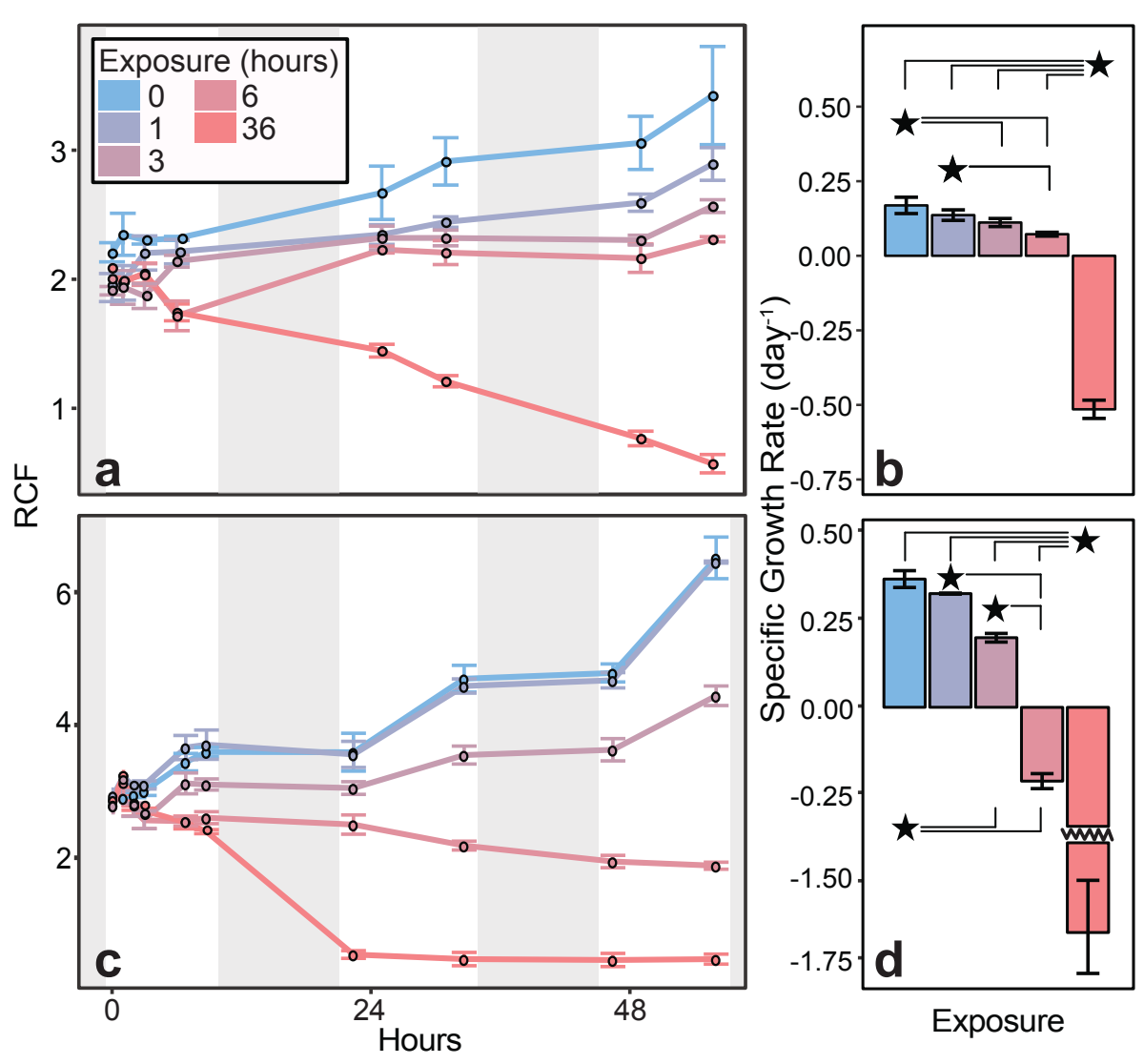

Figure 4: Effects of temperature and light exposure time on cultures of the novel Chaetoceros $s p$. isolate exposed to $638 \mu \mathrm{mol}$ photons $\mathrm{m}^{-2} \mathrm{sec}^{-1}$, the highest incident light level recorded in 41 years of data from its isolation location. a) Fluorescence (RCF) and b) specific growth rates $\left(\mathrm{d}^{-1}\right)$ of cultures grown at $4{ }^{\circ} \mathrm{C}$ under extreme light exposure. c) and d) show the same parameters for cultures grown under extreme light at $16^{\circ} \mathrm{C}$. In panels a \& c, periods of darkness are shown as grey bands. b \& $\mathbf{d}$ depict the growth rates for each exposure treatment after three days. All error bars indicate \pm 1 standard deviation. Stars and brackets show treatments that are significantly different by one-way ANOVA $(p<0.05)$. 
604

605

606

607

608

609
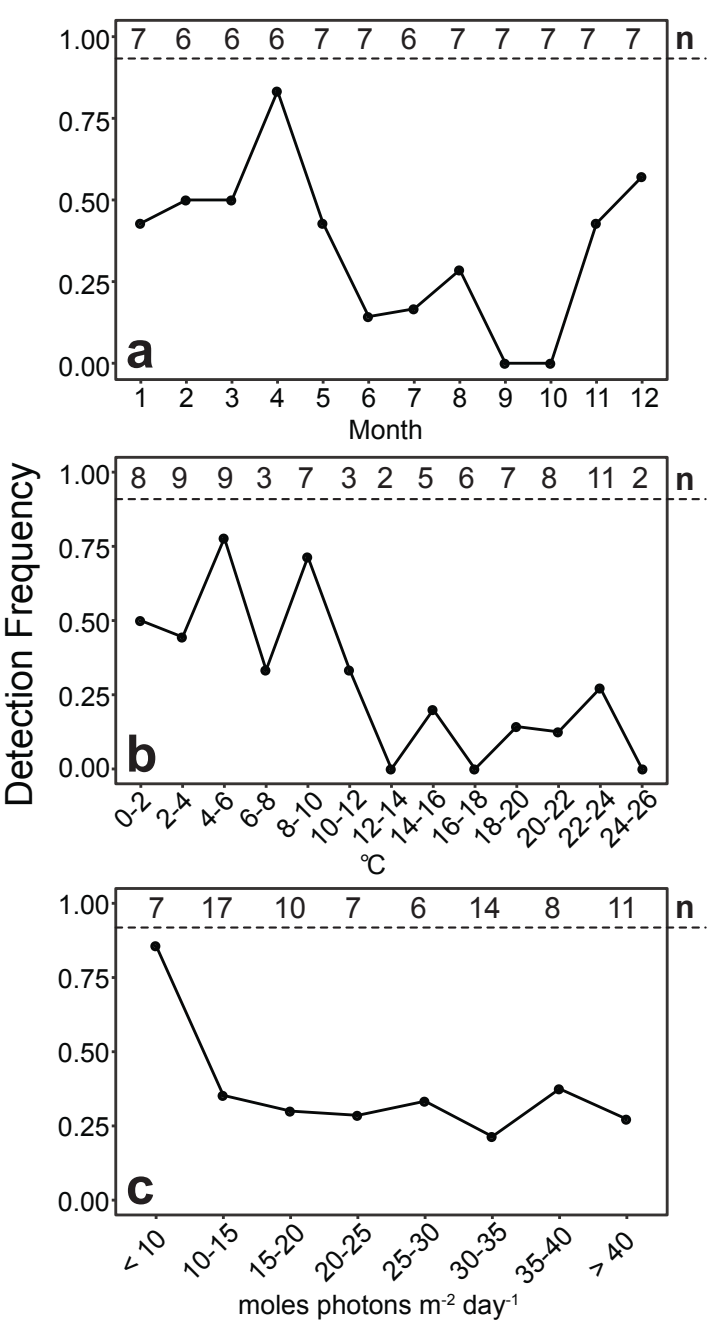

Figure 5: Probability of detection for the ASV matching the isolate described in this study in five years of $18 \mathrm{~S}$ rRNA gene amplicon data by a) month, b) temperature, and c) seven-day average of photons received per square meter. The number of samples falling within each category is shown along the top of each graph. 


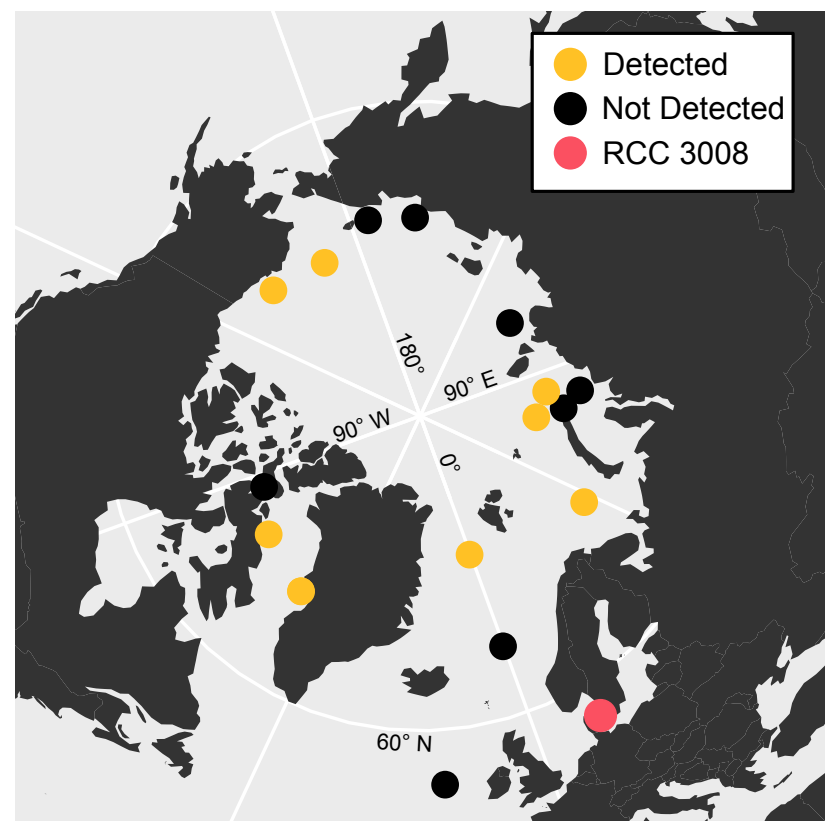

Figure 6: Combined relative abundance of two ASVs in the Tara Polar Ocean Circle stations that were $100 \%$ match to the V9 region of the $18 \mathrm{~S}$ rRNA gene sequence recovered from the diatom isolate presented in this study. The red dot shows the isolation location of a diatom in the Roscoff Culture Collection whose 18S rRNA gene is a close match to this isolate. 
618 Table 1: Thermal performance curve (TPC) parameters calculated at three light intensities.

619 Standard deviations are shown where available, within parenthesis.

620

\begin{tabular}{ccccccc}
$\begin{array}{c}\boldsymbol{\mu} \text { mol photons } \\
\mathbf{m}^{-2} \mathbf{s e c}^{-1}\end{array}$ & Width & Tmin & Tmax & Topt & $\boldsymbol{\mu M a x}$ & $\mathbf{r}^{\mathbf{2}}$ \\
\hline 15 & 25.7 & -2.0 & 23.7 & $13.7( \pm 0.87)$ & $0.16( \pm 0.01)$ & 0.87 \\
30 & 27.0 & -2.0 & 25.0 & $17.2( \pm 0.86)$ & $0.28( \pm 0.02)$ & 0.75 \\
50 & 23.2 & 1.5 & 24.7 & $15.2( \pm 1.17)$ & $0.25( \pm 0.02)$ & 0.71
\end{tabular}

621

622

623 